\title{
MAGIC detection of Geminga: an Inverse Compton tail?
}

\section{Giovanni Ceribella, ${ }^{a, *}$ Marcos López-Moya $^{b}$ and Razmik Mirzoyan ${ }^{a}$ on behalf of the MAGIC Collaboration}

(a complete list of authors can be found at the end of the proceedings)

${ }^{a}$ Max-Planck-Institut für Physik

Föhringer Ring 6, 80805 München, Germany

${ }^{b}$ Universidad Complutense de Madrid, IPARCOS Institute and EMFTEL Department

Ciudad Universitaria, Plaza Ciencias 1, 28040 Madrid, Spain

E-mail: ceribell@mpp.mpg.de

We report the detection of pulsed emission from the Geminga pulsar (PSR J0633+1746) with the MAGIC telescopes. After the Crab and Vela pulsars, Geminga is the third pulsar detected in the very-high-energy domain, and its estimated age of $340 \mathrm{ky}$ makes it the oldest one. The spectrum derived by MAGIC extends from $15 \mathrm{GeV}$ to $75 \mathrm{GeV}$ and can be modeled by a power-law function with spectral index $\Gamma=5.62 \pm 0.54$. Joint fits to MAGIC and Fermi-LAT data disfavour the existence of a sub-exponential cut-off in this energy range. Our results are discussed in the framework of the outer gap pulsar model. The measured power-law emission can be interpreted as the transition from curvature radiation to inverse Compton (IC) scattering of charges accelerated in the northern outer gap. The IC component is expected to continue towards higher energies. These results have been published in Acciari et al. 2020 [1].

$37^{\text {th }}$ International Cosmic Ray Conference (ICRC 2021)

July 12th - 23rd, 2021

Online - Berlin, Germany

\footnotetext{
*Presenter
} 


\section{Introduction}

Since the launch of the Fermi-LAT space telescope the number of known gamma-ray pulsars has increased dramatically. With more than 270 detections, pulsars are now the largest class of $\mathrm{GeV}$ sources in our galaxy. The spectrum of most Fermi-LAT pulsars exhibits a sharp cutoff at a few $\mathrm{GeVs}$, making them hard to detect with ground-based Cherenkov telescopes. Actually, apart from Geminga, only the Crab [2] and Vela [3] pulsars have been firmly detected from ground. In the case of $\mathrm{Crab}$, the emission continues up to $\mathrm{TeV}$ energies following a power-law function without any evidence of a cut-off [4]. The origin of the high-energy emission in pulsars is still unclear. Therefore, finding more pulsars at high energies is one of the main goals of Cherenkov telescopes.

Similarly to Crab and Vela, Geminga (PSR J0633+1746) is one of the strongest sources in the $\mathrm{GeV}$ sky. But unlike them, Geminga is much older and lacks radio emission. The first Geminga spectrum obtained by Fermi-LAT already showed a deviation from an exponential cutoff above 10 $\mathrm{GeV}$ [5]. However, subsequent attempts to detect Geminga at higher energies with VERITAS [6] and MAGIC [7] were unsuccessful. This made it clear that further technical developments were needed in order to detect Geminga with Cherenkov telescopes.

\section{Observations}

The MAGIC telescopes are a set of two 17-meter diameter Cherenkov telescopes located on the Canary Island of La Palma. In recent years we have significantly improved their performance below $100 \mathrm{GeV}$ with the introduction of a novel trigger system, the Sum-Trigger-II, which halves the energy threshold of the telescopes. Technical details on the implementation of the Sum-Trigger-II are discussed in [8].

Observations of the Geminga pulsar with MAGIC were carried out using the Sum-Trigger-II system between 2017 and 2019. A total of $80 \mathrm{~h}$ of good quality data were collected. A dedicated analysis pipepline was developed to improve the analysis of low-energy events, in which the calibration and the image cleaning are performed in an iterative procedure. The analysis includes the use of dedicated Monte Carlo simulations of gamma-ray showers to properly account for the effect of the geomagnetic field at tens of GeV.

MAGIC observations were combined with 11 years of Fermi-LAT data. Geminga ephemeris were obtained using Fermi-LAT data [9] and used to phase-fold MAGIC events. The two peaks visible in the Fermi-LAT light curve were fitted to symmetric Gaussian functions to obtain the expected phase signal regions for the MAGIC analysis.

\section{Results}

The Geminga light curve obtained by MAGIC above $15 \mathrm{GeV}$ is shown in Fig. 1. Emission from the second peak, P2, is detected at a significance level of $6.25 \sigma$. No significant signal is found in MAGIC data in the P1 phase region. The MAGIC spectrum in the energy range $15-75 \mathrm{GeV}$ is well-represented by a power-law function with spectral index $\Gamma=5.62 \pm 0.54$ [1]. The spectrum merges smoothly with the one measured by Fermi-LAT and is in agreement with the upper limits previously reported by MAGIC [7]. 


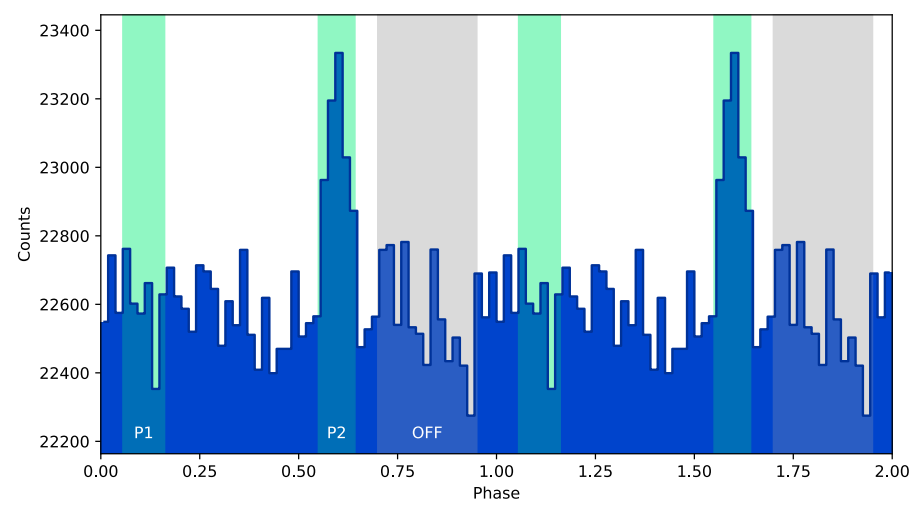

Figure 1: Geminga light curve measured by MAGIC above $15 \mathrm{GeV}$. Two rotation cycles are shown for clarity. The green-shaded regions highlight the phase intervals corresponding to the P1 and P2 peaks.

To test for the presence of an exponential cut-off in the combined MAGIC and Fermi-LAT spectrum, expected in classical pulsar models, we performed a joint fit to a power-law function with an exponential cut-off. A pure exponential cut-off is rejected at a significance level of $18 \sigma$. Also, the sub-exponential cut-off case is disfavoured at $3.6 \sigma$.

We compared our observational results with simulations of the Geminga pulsar in the framework of the outer gap model [10]. The measured emission from P2 by MAGIC requires the angle between the observer's line of sight and the pulsar rotation axis to be nearly $90^{\circ}$. In this case, the detected gamma rays would be produced in the northern outer gap via curvature radiation, for photons below $\sim 40 \mathrm{GeV}$, and by Inverse Compton scattering for the higher end of the reported spectrum.

\section{Conclusions}

We have presented the detection of pulsed gamma-ray emission from the Geminga pulsar with the MAGIC telescopes. Data were taken using the Sum-Trigger-II system developed for reducing the energy threshold of the telescopes. The emission detected above $15 \mathrm{GeV}$ coincides in phase with the position of the second emission peak of the pulsar. The measured spectrum up to $75 \mathrm{GeV}$ merges smoothly with the one measured at lower energies by Fermi-LAT, and is well described by a power law with spectral index $\Gamma=5.62 \pm 0.54$. Simulations of the outer gap pulsar model indicate that electrons accelerated in northern outer gap would be responsible for the most energetic gamma rays detected in the Geminga pulsar.

\section{Acknowledgements}

We would like to thank the Instituto de Astrofísica de Canarias for the excellent working conditions at the Observatorio del Roque de los Muchachos in La Palma. The financial support of the German BMBF, MPG and HGF; the Italian INFN and INAF; the Swiss National Fund SNF; the ERDF under the Spanish Ministerio de Ciencia e Innovación (MICINN) (PID2019-104114RB-C31, PID2019-104114RB-C32, PID2019-104114RB-C33, PID2019-105510GB-C31,PID2019-107847RBC41, PID2019-107847RB-C42, PID2019-107847RB-C44, PID2019-107988GB-C22); the Indian Department of Atomic Energy; the Japanese ICRR, the University of Tokyo, JSPS, and MEXT; the 
Bulgarian Ministry of Education and Science, National RI Roadmap Project DO1-400/18.12.2020 and the Academy of Finland grant nr. 320045 is gratefully acknowledged. This work was also supported by the Spanish Centro de Excelencia "Severo Ochoa" (SEV-2016-0588, SEV-20170709, CEX2019-000920-S), the Unidad de Excelencia "María de Maeztu" (CEX2019-000918-M, MDM-2015-0509-18-2) and by the CERCA program of the Generalitat de Catalunya; by the Croatian Science Foundation (HrZZ) Project IP-2016-06-9782 and the University of Rijeka Project 13.12.1.3.02; by the DFG Collaborative Research Centers SFB823/C4 and SFB876/C3; the Polish National Research Centre grant UMO-2016/22/M/ST9/00382; and by the Brazilian MCTIC, CNPq and FAPERJ.

\section{References}

[1] V. A. Acciari et al. (MAGIC Coll.), "Detection of the Geminga pulsar with MAGIC hints at a power-law tail emission beyond 15 GeV," Astron. Astrophys. 643 (2020) L14 doi:10.1051/0004-6361/202039131 [arXiv:2011.10412 [astro-ph.HE]].

[2] E. Aliu, et al. (MAGIC Coll.), Science 322 (2016) 1221

[3] H. Abdalla et al. (H.E.S.S. Coll.), Astronomy \& Astrophysic 620 (2018) A66

[4] S. Ansoldi et al. (MAGIC Coll.), Astronomy \& Astrophysics 585 (2016) A133

[5] A. Abdo (Fermi-LAT Coll.) Astrop. Journal 720 (2010) 272

[6] E. Aliu. et al. (VERITAS Coll.), Astrop. Journal 800 (2015) 61

[7] M. L. Ahnen et al. (MAGIC Coll.), Astronomy \& Astrophysics 591 (2016) A138

[8] F. Dazzi et al., IEEE Transactions on Nuclear Science $\mathbf{6 8}$ (2021) 7 doi:10.1109/TNS.2021.3079262

[9] M. Kerr, P. S. Ray, S. Johnston, R. M. Shannon \& F. Camilo, Astrop. Journal 814 (2015) 128

[10] K. Hirotani Astrop. Journal 766 (2013) 98 


\section{Full Authors List: MAGIC Collaboration}

V. A. Acciari ${ }^{1}$, S. Ansoldi ${ }^{2,41}$, L. A. Antonelli ${ }^{3}$, A. Arbet Engels $^{4}$, M. Artero ${ }^{5}$, K. Asano $^{6}$, D. Baack ${ }^{7}$, A. Babić ${ }^{8}$, A. Baquero ${ }^{9}$, U. Barres de Almeida ${ }^{10}$, J. A. Barrio ${ }^{9}$, I. Batković ${ }^{11}$, J. Becerra González ${ }^{1}$, W. Bednarek ${ }^{12}$, L. Bellizzi ${ }^{13}$, E. Bernardini ${ }^{14}$, M. Bernardos ${ }^{11}$, A. Berti ${ }^{15}$, J. Besenrieder ${ }^{15}$, W. Bhattacharyya ${ }^{14}$, C. Bigongiari ${ }^{3}$, A. Biland ${ }^{4}$, O. Blanch ${ }^{5}$, H. Bökenkamp ${ }^{7}$, G. Bonnoli ${ }^{16}$, Ž. Bošnjak $^{8}$, G. Busetto ${ }^{11}$, R. Carosi ${ }^{17}$, G. Ceribella ${ }^{15}$, M. Cerruti ${ }^{18}$, Y. Chai ${ }^{15}$, A. Chilingarian ${ }^{19}$, S. Cikota ${ }^{8}$, S. M. Colak ${ }^{5}$, E. Colombo ${ }^{1}$, J. L. Contreras ${ }^{9}$, J. Cortina ${ }^{20}$, S. Covino ${ }^{3}$, G. D’Amico ${ }^{15,42}$, V. D’Elia ${ }^{3}$, P. Da Vela ${ }^{17,43}$, F. Dazzi ${ }^{3}$, A. De Angelis ${ }^{11}$, B. De Lotto ${ }^{2}$, M. Delfino ${ }^{5,44}$, J. Delgado ${ }^{5,44}$, C. Delgado Mendez ${ }^{20}$, D. Depaoli ${ }^{21}$, F. Di Pierro ${ }^{21}$, L. Di Venere ${ }^{22}$, E. Do Souto Espiñeira ${ }^{5}$, D. Dominis Prester $^{23}$, A. Donini ${ }^{2}$, D. Dorner ${ }^{24}$, M. Doro ${ }^{11}$, D. Elsaesser ${ }^{7}$, V. Fallah Ramazani ${ }^{25,45}$, A. Fattorini ${ }^{7}$, M. V. Fonseca ${ }^{9}$, L. Font ${ }^{26}$, C. Fruck ${ }^{15}$, S. Fukami ${ }^{6}$, Y. Fukazawa ${ }^{27}$, R. J. García López ${ }^{1}$, M. Garczarczyk ${ }^{14}$, S. Gasparyan ${ }^{28}$, M. Gaug ${ }^{26}$, N. Giglietto ${ }^{22}$, F. Giordano ${ }^{22}$, P. Gliwny ${ }^{12}$, N. Godinović ${ }^{29}$, J. G. Green ${ }^{3}$, D. Green ${ }^{15}$, D. Hadasch ${ }^{6}$, A. Hahn ${ }^{15}$, L. Heckmann ${ }^{15}$, J. Herrera ${ }^{1}$, J. Hoang ${ }^{9,46}$, D. Hrupec ${ }^{30}$, M. Hütten ${ }^{15}$, T. Inada ${ }^{6}$, K. Ishio ${ }^{12}$, Y. Iwamura ${ }^{6}$, I. Jiménez Martínez ${ }^{20}$, J. Jormanainen ${ }^{25}$, L. Jouvin ${ }^{5}$, M. Karjalainen ${ }^{1}$, D. Kerszberg ${ }^{5}$, Y. Kobayashi ${ }^{6}$, H. Kubo ${ }^{31}$, J. Kushida ${ }^{32}$, A. Lamastra ${ }^{3}$, D. Lelas ${ }^{29}$, F. Leone ${ }^{3}$, E. Lindfors ${ }^{25}$, L. Linhoff ${ }^{7}$, S. Lombardi ${ }^{3}$, F. Longo ${ }^{2,47}$, R. López-Coto ${ }^{11}$, M. López-Moya ${ }^{9}$, A. López-Oramas ${ }^{1}$, S. Loporchio ${ }^{22}$, B. Machado de Oliveira Fraga $^{10}$, C. Maggio ${ }^{26}$, P. Majumdar ${ }^{33}$, M. Makariev ${ }^{34}$, M. Mallamaci ${ }^{11}$, G. Maneva ${ }^{34}$, M. Manganaro ${ }^{23}$, K. Mannheim ${ }^{24}$, L. Maraschi ${ }^{3}$, M. Mariotti ${ }^{11}$, M. Martínez ${ }^{5}$, D. Mazin ${ }^{6,48}$, S. Menchiari ${ }^{13}$, S. Mender ${ }^{7}$, S. Mićanović23, D. Miceli ${ }^{2,49}$, T. Miener ${ }^{9}$, J. M. Miranda ${ }^{13}$, R. Mirzoyan ${ }^{15}$, E. Molina ${ }^{18}$, A. Moralejo ${ }^{5}$, D. Morcuende ${ }^{9}$, V. Moreno ${ }^{26}$, E. Moretti ${ }^{5}$, T. Nakamori ${ }^{35}$, L. Nava ${ }^{3}$, V. Neustroev ${ }^{36}$, C. Nigro $^{5}$, K. Nilsson ${ }^{25}$, K. Nishijima ${ }^{32}$, K. Noda $^{6}$, S. Nozaki ${ }^{31}$, Y. Ohtani' ${ }^{6}$, T. Oka ${ }^{31}$, J. Otero-Santos ${ }^{1}$, S. Paiano ${ }^{3}$, M. Palatiello ${ }^{2}$, D. Paneque ${ }^{15}$, R. Paoletti ${ }^{13}$, J. M. Paredes ${ }^{18}$, L. Pavletić ${ }^{23}$, P. Peñil ${ }^{9}$, M. Persic ${ }^{2,50}$, M. Pihet ${ }^{15}$, P. G. Prada Moroni ${ }^{17}$, E. Prandini ${ }^{11}$, C. Priyadarshi ${ }^{5}$, I. Puljak ${ }^{29}$, W. Rhode ${ }^{7}$, M. Ribó ${ }^{18}$, J. Rico ${ }^{5}$, C. Righi ${ }^{3}$, A. Rugliancich ${ }^{17}$, N. Sahakyan ${ }^{28}$, T. Saito ${ }^{6}$, S. Sakurai ${ }^{6}$, K. Satalecka ${ }^{14}$, F. G. Saturni ${ }^{3}$, B. Schleicher ${ }^{24}$, K. Schmidt $^{7}$, T. Schweizer ${ }^{15}$, J. Sitarek ${ }^{12}$, I. Šnidarić ${ }^{37}$, D. Sobczynska ${ }^{12}$, A. Spolon ${ }^{11}$, A. Stamerra ${ }^{3}$, J. Strišković ${ }^{30}$, D. Strom ${ }^{15}$, M. Strzys ${ }^{6}$, Y. Suda ${ }^{27}$, T. Surić ${ }^{37}$, M. Takahashi ${ }^{6}$, R. Takeishi ${ }^{6}$, F. Tavecchio ${ }^{3}$, P. Temnikov ${ }^{34}$, T. Terzić ${ }^{23}$, M. Teshima ${ }^{15,51}$, L. Tosti ${ }^{38}$, S. Truzzi $^{13}$, A. Tutone ${ }^{3}$, S. Ubach ${ }^{26}$, J. van Scherpenberg ${ }^{15}$, G. Vanzo $^{1}$, M. Vazquez Acosta ${ }^{1}$, S. Ventura ${ }^{13}$, V. Verguilov ${ }^{34}$, C. F. Vigorito ${ }^{21}$, V. Vitale ${ }^{39}$, I. Vovk $^{6}$, M. Will ${ }^{15}$, C. Wunderlich ${ }^{13}$, T. Yamamoto $^{40}$, and D. Zarić 29

${ }^{1}$ Instituto de Astrofísica de Canarias and Dpto. de Astrofísica, Universidad de La Laguna, E-38200, La Laguna, Tenerife, Spain ${ }^{2}$ Università di Udine and INFN Trieste, I-33100 Udine, Italy ${ }^{3}$ National Institute for Astrophysics (INAF), I-00136 Rome, Italy ${ }^{4}$ ETH Zürich, CH-8093 Zürich, Switzerland ${ }^{5}$ Institut de Física d'Altes Energies (IFAE), The Barcelona Institute of Science and Technology (BIST), E-08193 Bellaterra (Barcelona), Spain ${ }^{6}$ Japanese MAGIC Group: Institute for Cosmic Ray Research (ICRR), The University of Tokyo, Kashiwa, 277-8582 Chiba, Japan ${ }^{7}$ Technische Universität Dortmund, D-44221 Dortmund, Germany ${ }^{8}$ Croatian MAGIC Group: University of Zagreb, Faculty of Electrical Engineering and Computing (FER), 10000 Zagreb, Croatia ${ }^{9}$ IPARCOS Institute and EMFTEL Department, Universidad Complutense de Madrid, E-28040 Madrid, Spain ${ }^{10}$ Centro Brasileiro de Pesquisas Físicas (CBPF), 22290-180 URCA, Rio de Janeiro (RJ), Brazil ${ }^{11}$ Università di Padova and INFN, I-35131 Padova, Italy ${ }^{12}$ University of Lodz, Faculty of Physics and Applied Informatics, Department of Astrophysics, 90-236 Lodz, Poland ${ }^{13}$ Università di Siena and INFN Pisa, I-53100 Siena, Italy ${ }^{14}$ Deutsches Elektronen-Synchrotron (DESY), D-15738 Zeuthen, Germany ${ }^{15}$ Max-Planck-Institut für Physik, D-80805 München, Germany ${ }^{16}$ Instituto de Astrofísica de Andalucía-CSIC, Glorieta de la Astronomía s/n, 18008, Granada, Spain ${ }^{17}$ Università di Pisa and INFN Pisa, I-56126 Pisa, Italy ${ }^{18}$ Universitat de Barcelona, ICCUB, IEEC-UB, E-08028 Barcelona, Spain ${ }^{19}$ Armenian MAGIC Group: A. Alikhanyan National Science Laboratory, 0036 Yerevan, Armenia ${ }^{20}$ Centro de Investigaciones Energéticas, Medioambientales y Tecnológicas, E-28040 Madrid, Spain ${ }^{21}$ INFN MAGIC Group: INFN Sezione di Torino and Università degli Studi di Torino, I-10125 Torino, Italy 22 INFN MAGIC Group: INFN Sezione di Bari and Dipartimento Interateneo di Fisica dell'Università e del Politecnico di Bari, I-70125 Bari, Italy ${ }^{23}$ Croatian MAGIC Group: University of Rijeka, Department of Physics, 51000 Rijeka, Croatia ${ }^{24}$ Universität Würzburg, D-97074 Würzburg, Germany ${ }^{25}$ Finnish MAGIC Group: Finnish Centre for Astronomy with ESO, University of Turku, FI-20014 Turku, Finland ${ }^{26}$ Departament de Física, and CERES-IEEC, Universitat Autònoma de Barcelona, E-08193 Bellaterra, Spain 27 Japanese MAGIC Group: Physics Program, Graduate School of Advanced Science and Engineering, Hiroshima University, 7398526 Hiroshima, Japan ${ }^{28}$ Armenian MAGIC Group: ICRANet-Armenia at NAS RA, 0019 Yerevan, Armenia ${ }^{29}$ Croatian MAGIC Group: University of Split, Faculty of Electrical Engineering, Mechanical Engineering and Naval Architecture (FESB), 21000 Split, Croatia ${ }^{30}$ Croatian MAGIC Group: Josip Juraj Strossmayer University of Osijek, Department of Physics, 31000 Osijek, Croatia ${ }^{31}$ Japanese MAGIC Group: Department of Physics, Kyoto University, 606-8502 Kyoto, Japan ${ }^{32}$ Japanese MAGIC Group: Department of Physics, Tokai University, Hiratsuka, 259-1292 Kanagawa, Japan ${ }^{33}$ Saha Institute of Nuclear Physics, HBNI, 1/AF Bidhannagar, Salt Lake, Sector-1, Kolkata 700064, India ${ }^{34}$ Inst. for Nucl. Research and Nucl. Energy, Bulgarian Academy of Sciences, BG-1784 Sofia, Bulgaria ${ }^{35}$ Japanese MAGIC Group: Department of Physics, Yamagata University, Yamagata 990-8560, Japan ${ }^{36}$ Finnish MAGIC Group: Astronomy Research Unit, University of Oulu, FI-90014 Oulu, Finland ${ }^{37}$ Croatian MAGIC Group: Ruđer Bošković Institute, 10000 Zagreb, Croatia ${ }^{38}$ INFN MAGIC Group: INFN Sezione di Perugia, I-06123 Perugia, Italy ${ }^{39}$ INFN MAGIC Group: INFN Roma Tor Vergata, I-00133 Roma, Italy ${ }^{40}$ Japanese MAGIC Group: Department of Physics, Konan University, Kobe, Hyogo 658-8501, Japan ${ }^{41}$ also at International Center for Relativistic Astrophysics (ICRA), Rome, Italy ${ }^{42}$ now at Department for Physics and Technology, University of Bergen, NO-5020, Norway ${ }^{43}$ now at University of Innsbruck ${ }^{44}$ also at Port d'Informació Científica (PIC), E-08193 Bellaterra (Barcelona), Spain ${ }^{45}$ now at Ruhr-Universität Bochum, Fakultät für Physik und Astronomie, Astronomisches Institut (AIRUB), 44801 Bochum, Germany ${ }^{46}$ now at Department of Astronomy, University of California Berkeley, Berkeley CA 94720 47 also at Dipartimento di Fisica, Università di Trieste, I-34127 Trieste, Italy ${ }^{48}$ Max-Planck-Institut für Physik, D-80805 München, Germany ${ }^{49}$ now at Laboratoire d'Annecy de Physique des Particules (LAPP), CNRS-IN2P3, 74941 Annecy Cedex, France ${ }^{50}$ also at 
INAF Trieste and Dept. of Physics and Astronomy, University of Bologna, Bologna, Italy ${ }^{51}$ Japanese MAGIC Group: Institute for Cosmic Ray Research (ICRR), The University of Tokyo, Kashiwa, 277-8582 Chiba, Japan 